\title{
Transmissible cytotoxicity of multiple myeloma cells by cord blood-derived NK cells is mediated by vesicle trafficking
}

\author{
B Martin-Antonio ${ }^{1,2}$, A Najjar ${ }^{3}$, SN Robinson ${ }^{1}$, C Chew ${ }^{1}$, S Li $^{1}$, E Yvon ${ }^{1}$, MW Thomas ${ }^{1}$, I Mc Niece ${ }^{1}$, R Orlowski ${ }^{4}$, C Muñoz-Pinedo ${ }^{5}$, \\ C Bueno ${ }^{6}$, P Menendez ${ }^{6,7}$, C Fernández de Larrea ${ }^{2}$, A Urbano-Ispizua ${ }^{2}$, EJ Shpall ${ }^{1}$ and N Shah ${ }^{1}$
}

\begin{abstract}
Natural killer cells (NK) are important effectors of anti-tumor immunity, activated either by the downregulation of HLA-I molecules on tumor cells and/or the interaction of NK-activating receptors with ligands that are overexpressed on target cells upon tumor transformation (including NKG2D and NKP30). NK kill target cells by the vesicular delivery of cytolytic molecules such as Granzyme-B and Granulysin activating different cell death pathways, which can be Caspase-3 dependent or Caspase-3 independent. Multiple myeloma (MM) remains an incurable neoplastic plasma-cell disorder. However, we previously reported the encouraging observation that cord blood-derived NK (CB-NK), a new source of NK, showed anti-tumor activity in an in vivo murine model of MM and confirmed a correlation between high levels of NKG2D expression by MM cells and increased efficacy of CB-NK in reducing tumor burden. We aimed to characterize the mechanism of CB-NK-mediated cytotoxicity against MM cells. We show a Caspase-3- and Granzyme-B-independent cell death, and we reveal a mechanism of transmissible cell death between cells, which involves lipid-protein vesicle transfer from CB-NK to MM cells. These vesicles are secondarily transferred from recipient MM cells to neighboring MM cells amplifying the initial CB-NK cytotoxicity achieved. This indirect cytotoxicity involves the transfer of NKG2D and NKP30 and leads to lysosomal cell death and decreased levels of reactive oxygen species in MM cells. These findings suggest a novel and unique mechanism of CB-NK cytotoxicity against MM cells and highlight the importance of lipids and lipid transfer in this process. Further, these data provide a rationale for the development of CB-NK-based cellular therapies in the treatment of MM.
\end{abstract}

Cell Death and Differentiation (2015) 22, 96-107; doi:10.1038/cdd.2014.120; published online 29 August 2014

Natural killer cells (NK) are important effectors of anti-tumor immunity of the immune system. They can be activated by inhibition of killer cell immunoglobulin (lg)-like receptor (KIR) receptors on NK due to downregulation of HLA-I on tumor cells or by the interaction of NK-activating receptors with ligands that are overexpressed on target cells. These receptors include NKG2D and the family of NK cytotoxicity receptors (NKP30, NKP44, NKP46), ${ }^{1}$ which can be on the cell surface and in the endocytic compartment ${ }^{2}$ from where they traffic to tumor cells in exosomes to exert cytotoxicity. ${ }^{3}$

NK deliver cytolytic molecules such as Granzyme-B (GrB) and Granulysin to target cells. GrB induces Caspase-3-dependent apoptotic cell death with reactive oxygen species (ROS) generation. ${ }^{4}$ Alternatively, Granulysin activates Caspase-3dependent cell death through ROS generation ${ }^{5,6}$ and Caspase-3-independent cell death via endoplasmic reticulum (ER) stress $^{7}$ or lysosomal cell death through release of cathepsins. ${ }^{8}$

Multiple myeloma (MM) is a plasma-cell disorder characterized by clonal proliferation of malignant plasma-cells in the bone marrow (BM) and monoclonal protein in the blood or urine. $^{9,10}$ Plasma cells synthesize large quantities of Igs, which are folded in the ER. An excess of Ig synthesis causes a failure in this folding process leading to the release of unfolded/misfolded Igs. ${ }^{11}$ These Igs are degraded by intracellular protein degradation pathways, including the ubiquitinproteasome system and autophagy. Proteasome inhibitors (PIs) are potent anti-MM agents, ${ }^{12}$ which block the protein degradation process in MM cells leading to ER stressmediated cell death. ${ }^{13,14}$ An excess of Pl-induced ER stress can increase autophagy ${ }^{15,16}$ leading to eventual refractory disease in some patients. ${ }^{17-19}$ Therefore new anti-MM strategies are needed.

Previously, we have demonstrated that cord blood-derived NK (CB-NK) show anti-tumor activity in an in vivo MM murine model $^{20}$ and observed that the expression of NKG2D by MM tumor cells correlated with lower tumor burden following CB-NK cellular therapy. Here we characterize the CB-NKmediated cytotoxicity against MM cells and observe a

\footnotetext{
${ }^{1}$ Department of Stem Cell Transplantation and Cellular Therapy, The University of Texs M.D. Anderson Cancer Center, Houston, TX, USA; ${ }^{2}$ Department of Hematology, Hospital Clinic, IDIBAPS, Josep Carreras Leukaemia Research Institute/University of Barcelona, Barcelona, Spain; ${ }^{3}$ Department of Cancer Systems Imaging, The University of Texs M.D. Anderson Cancer Center, Houston, TX, USA; ${ }^{4}$ Department of Lymphoma/Myeloma, The University of Texas M.D. Anderson Cancer Center, Houston, TX, USA; ${ }^{5}$ Cell Death Regulation Group, Bellvitge Biomedical Research Institute (IDIBELL), Barcelona, Spain; ${ }^{6}$ Josep Carreras Leukemia Research Institute and Cell Therapy Program of the School of Medicine, University of Barcelona, Barcelona, Spain and ${ }^{7}$ Institució Catalana de Recerca i Estudis Avançats (ICREA), Barcelona, Spain

${ }^{*}$ Corresponding author: B Martin-Antonio, Department of Hematology, Hospital Clinic, IDIBAPS, Josep Carreras Leukaemia Research Institute, Facultat de Medicina, University of Barcelona, Carrer Casanova 143, Barcelona 08036, Spain. Tel: + 935572810; Fax: + 34 934035263; E-mail: bmartina @ clinic.ub.es Abbreviations: NK, natural killer cells; CB-NK, cord blood-derived NK cells; MM, multiple myeloma; ROS, reactive oxygen species; ER, endoplasmic reticulum; GrB, Granzyme-B

Received 05.2.14; revised 02.7.14; accepted 09.7.14; Edited by JP Medema; published online 29.8.14
} 
Caspase-3- and Gr-B-independent cell death and reveal a mechanism of transmissible cell death between cells that involves lipid-protein vesicle transfer from CB-NK to MM cells. These vesicles are secondarily transferred from recipient MM cells to neighboring MM cells, thereby amplifying the initial CB-NK cytotoxicity achieved. This indirect cytotoxicity involves the transfer of NKG2D and NKP30 and leads to lysosomal cell death and reduced ROS levels in MM cells.

\section{Results}

NKG2D expression in MM cells after CB-NK treatment correlates with lower MM progression, and NKG2D and NKP30 contribute more to the cytotoxicity of MM cells than in K562 cells. We have previously shown that CB-NK exert anti-MM activity in a murine MM model. ${ }^{20}$ Immunodeficient mice were injected with the ARP1 MM cell line. MM cells were identified in the BM, spleen, lymph nodes (LN) and ovaries and demonstrated higher MFI for NKG2D in the BM, $\mathrm{LN}$ and ovaries from CB-NK-treated mice versus untreated mice (Supplementary Figures S1A-D and F-I). CB-NK in $\mathrm{CB}-\mathrm{NK}$ recipient mice were $\mathrm{CD}^{+}{ }^{+}, \mathrm{CD}^{-}, \mathrm{NKG}^{-} \mathrm{D}^{+}$and $\mathrm{CD}^{+}{ }^{+}$(Supplementary Figure S1J). In treated mice, MFI for NKG2D in MM cells in LN correlated inversely with tumor burden $(R=-0.886, P=0.04$; Supplementary Figure $S 1 \mathrm{~J})$, suggesting a transfer of NKG2D from CB-NK to MM cells with a beneficial effect.

As the presence of NKG2D in MM cells was associated with lower tumor burden, we investigated the cytotoxic role of NKG2D. We also examined NKP30, as it has an important role in the killing of MM cell lines, ${ }^{21}$ MICA/B (NKG2D ligand) and the synergistic effects of these receptors. We compared our results from ARP1 cells with K562 cells, where, in the absence of HLA-I, NK cytotoxicity is mediated by KIR receptors.

After blocking these receptors, we found a higher contribution of these in the killing of ARP1 versus K562 cells (Figures $1 \mathrm{a}-\mathrm{d})$. The greatest impact in ARP1 cells was mediated by NKP30 followed by NKG2D (Figures $1 \mathrm{~b}$ and d). At a 20:1 effector: target cell ratio, we observed a 33\% (NKG2D blocked), 49\% (NKP30 blocked) and 73\% (both blocked) reduction in ARP1 killing (Figure 1d) versus 4\% (NKG2D blocked), 3\% (NKP30 blocked) and 19\% (both blocked) reduction in K562 killing (Figure 1c). This effect was reproduced at high effector: target cell ratios (Figures 1c and $\mathrm{d}, P<0.0001)$. A similar pattern was observed with primary $\mathrm{MM} \mathrm{CD138}{ }^{+}$cells showing at $20: 1$ effector: target cell ratio a $26.6 \%$ (NKG2D blocked) and 51\% (NKP30 blocked) reduction in $\mathrm{CD}_{138}{ }^{+}$cell killing (Figures 1e-g). Moreover, in NK from MM patients (MM-NK) that showed lower cytotoxicity than CB-NK (Supplementary Figure S2A), NKG2D and NKP30 receptors did not impact NK-MM cytotoxicity (Supplementary Figure S2B), revealing this unique feature of CB-NK cytotoxicity and the superiority of CB-NK for MM treatment.

NKG2D and NKP30 are transferred into MM cells via lipid trafficking and the endocytic pathway. We reported an inverse correlation between NKG2D expression in MM-ARP1 cells and disease progression and demonstrated that NKG2D and NKP30 were involved in CB-NK cytotoxicity.
The trafficking of these receptors to MM cells was subsequently followed using confocal fluorescence microscopy. Cell trackers were used to label CB-NK and tumor cells. Tumor cells were labeled in blue using CMAC and CB-NK in green with bodipy-505/515. This has an affinity for neutral lipids and has a chloromethyl group that allows binding to membrane proteins, thereby labeling proteins and lipids. We observed that bodipy staining allowed us to follow the trafficking of contents from CB-NK to ARP1 cells (Figure 2, and Supplementary Movie S1).

Within $10 \mathrm{~min}$ of tumor cell/CB-NK co-culture, CB-NK transferred their contents to MM cells. This was via lipidprotein vesicles and larger volume vesicles (Figure 2a). Further, some of the lipid-protein vesicles trafficked between ARP1 cells after transfer from CB-NK (Figure 2b). Such transfer was not detected in the absence of CB-NK (Figure 2c). This trafficking also involved the immunological synapse, as demonstrated by actin co-staining (Figures $2 \mathrm{a}$ and b). Of note, MM-NK showed less intense immunological synapse versus MM cells and lower bodipy staining (Supplementary Figure S2C) in comparison to CB-NK supporting the finding of lower MM-NK cytotoxicity and indicating lower levels of lipids/cytotoxic vesicles in MM-NK. The stronger immunological synapses and bodipy staining, indicative of higher levels of lipids, were characteristic of more potent CB-NK.

Time-lapse in vivo imaging revealed that the intensity of the materials transferred to ARP1 cells by CB-NK increased with time as indicated by the increase in Bodipy (regions of interest (ROIs) 1-3 in Figures 2d and e). Also, after CB-NK transferred vesicles to ARP1 cells, these ARP1 cells could then transfer Bodipy-containing vesicles to neighboring ARP1 cells (ROIs 4 and 5 in Figures 2d, $f$ and $g$ and Supplementary Movie S1). This transfer was associated with membrane blebbing appearance (Figure $2 \mathrm{~g}$ ) indicative of apoptotic-like mechanisms. $^{22}$ Imaging revealed that, after $20 \mathrm{~min}$ of co-culture, NKG2D and NKP30 were detected in ARP1 cells (Figures 3ac), whereas they could not be detected in ARP1 cells that had not been with CB-NK (Figure $3 d$ ). After 3 days of co-culture, flow cytometry confirmed surface and intracellular presence of NKG2D and NKP30 in ARP1 cells (Figures 3e and f).

We investigated the role of the endocytic pathway in the NKG2D and NKP30 transfer to ARP1 cells. Cells uptake molecules from the extracellular environment by endocytosis. NK-activating receptors are continuously recycled and degraded by endocytic pathway. ${ }^{2}$ Once produced, NKactivating receptors are delivered to early endosomes (defined by EEA1). They are then either recycled to the plasma membrane (defined by Rab11) and exocytosed, or delivered to late endosomes (defined by Rab7), to be targeted to lysosomes for degradation. 2,23,24 We therefore analyzed whether NKG2D and NKP30 once transferred between cells entered the endocytic pathway in MM cells.

We observed that bodipy co-localized with NKG2D and NKP30 (column 3, Figure 4) confirming concomitant lipid and protein trafficking between cells. Co-localization of NKG2D and NKP30 with EEA1 (column 4, Figures 4a and d), Rab11 (column 4, Figures 4b and e) and Rab7 (column 4, Figures 4c and $\mathrm{f}$ ) indicated active trafficking of NKG2D and NKP30 through endocytic pathway involving lipid-protein trafficking. 

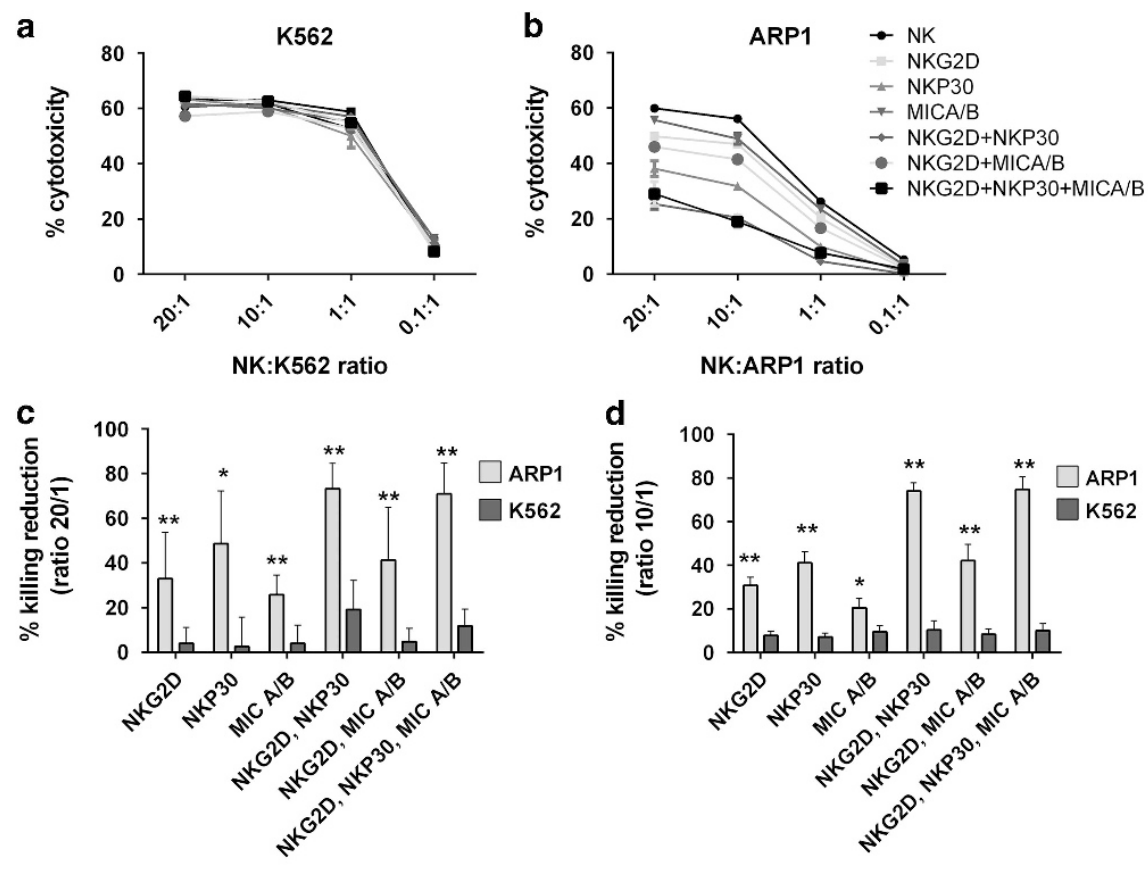

\section{NK receptor blocked}

e

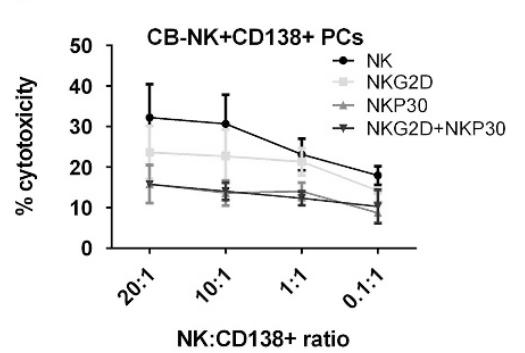

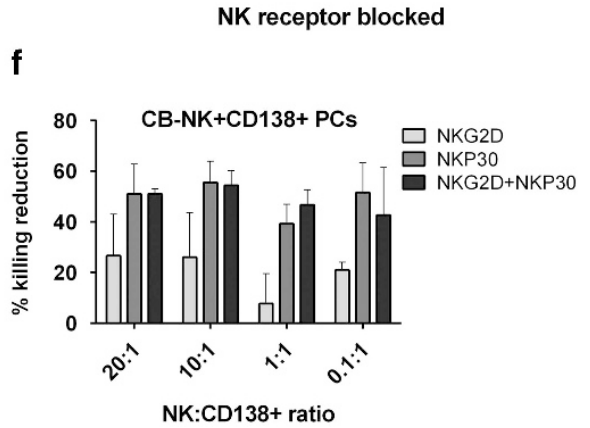

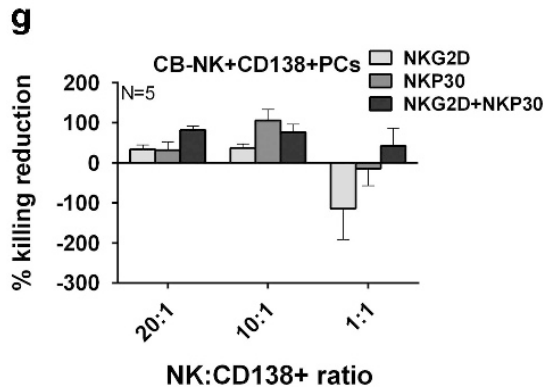

Figure 1 NKG2D and NKP30 contribute more to the cytotoxicity of MM cells than in K562 cells: (a and $\mathbf{b}$ ) Cytoxicity assays of CB-NK against K562 (a) and ARP1 (b) cells after blocking NKG2D, NKP30 in CB-NK and MICA/B in target cells. (c and d) Percentage of killing reduction obtained in panels $\mathbf{a}$ and $\mathbf{b}$ after blocking the receptors. Bars represent mean \pm S.E.M. values at a effector:target cell ratio 20:1 (c) and 10:1 (d) $(n=8)$. (e) CB-NK cytotoxicity assay versus primary CD138 ${ }^{+}$MM cells, adding CB-NK before and after blocking NKG2D, NKP30 and both together. (f) Percentage of killing reduction obtained in panel e after blocking the receptors. (g) Mean values of experiment shown in panels $\mathbf{e}$ and $\mathbf{f}$ with $\mathrm{CD} 138^{+}$cells from five MM patients. Bars represent mean \pm S.E.M. ${ }^{*} P \leq 0.05 .{ }^{* \star} P \leq 0.0001$

To confirm the importance of lipid-protein trafficking in the transfer of NKG2D and NKP30 and in CB-NK cytotoxicity, we analyzed the expression levels of NKG2D and NKP30 in target cells following preincubation of CB-NK with Filipin III (a lipid raft inhibitor) ${ }^{22,25}$ and performed cytotoxicty assays adding U18666A (a cholesterol synthesis and transport inhibitor ${ }^{26}$ ). Lipid rafts inhibition reduced the expression of NKG2D and NKP30 in ARP1 cells after CB-NK exposure (Figure $4 \mathrm{~g}$ ), providing evidence to support a role for lipidprotein trafficking in the transfer of NKG2D and NKP30. Cholesterol synthesis inhibition in CB-NK reduced cytotoxicity only versus ARP1 (Figures 4h and i); however, for K562 we observed higher cytotoxicity at 10:1 effector:target ratio, suggesting different roles for lipids in CB-NK cytotoxicity depending on the target cell. We confirmed in U266 and KMM1 MM cells the cytotoxic role of CB-NK-derived lipids (Supplementary Figure S3A). Interestingly, peripheral blood NK (PB-NK) of healthy individuals failed to show evidence of a cytotoxic role of lipids versus MM (Supplementary Figure S4A), suggesting another unique feature of CB-NK.
The mechanisms underlying CB-NK-mediated cytotoxicity differ between target cells. As we had evidence for different cytotoxic mechanisms depending on the target cells (MM versus K562) (Figure 1), other parameters were assessed. Upon interaction with target cells, NK degranulate releasing secretory lysosomes with $\mathrm{GrB}^{27}$ activating Caspase-3 in target cells with features of apoptosis, such as membrane blebbing and ROS generation. ${ }^{4}$ CB-NK showed less degranulation versus ARP1 than versus K562 cells (mean value: 7.6 versus $68 \%, P=0.029$ ) (Figures $5 a$ and $b$ ), which was confirmed for other MM cell lines (Supplementary Figure S3B). Further, we noted that GrB was transferred to ARP1 cells after contact (Figure $5 \mathrm{c}$ ). Interestingly, inhibition of GrB and Caspase-3 in NK-K562 and NK-ARP1 co-cultures reduced the killing of K562 cells by CB-NK as expected but increased the killing of ARP1 cells by CB-NK (Figures 5d and e). Inhibition of GrB and Caspase-3 at a 20:1 effector:target ratio yielded a reduction of $13 \%$ in CB-NK cytotoxicity against K562 cells but an increase of $12 \%$ in killing against ARP1 cells (Figures $5 e, P<0.05$ ). 

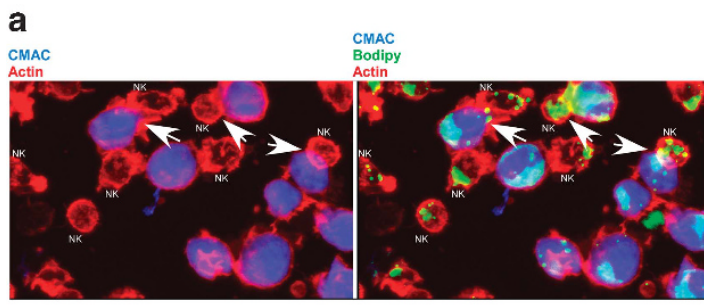

d

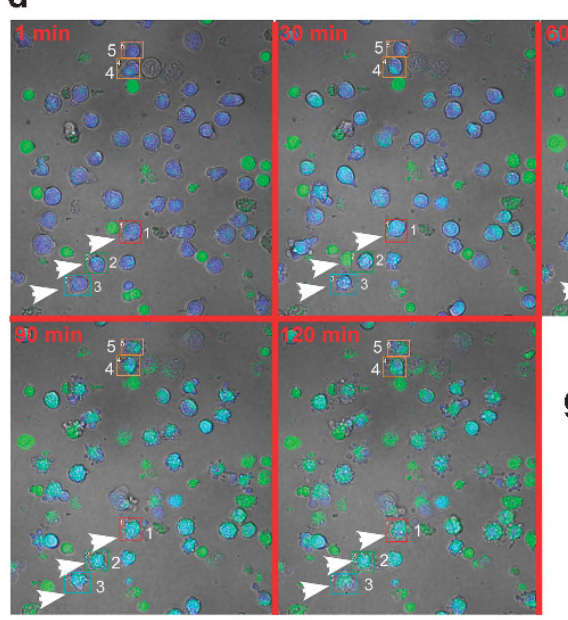

b
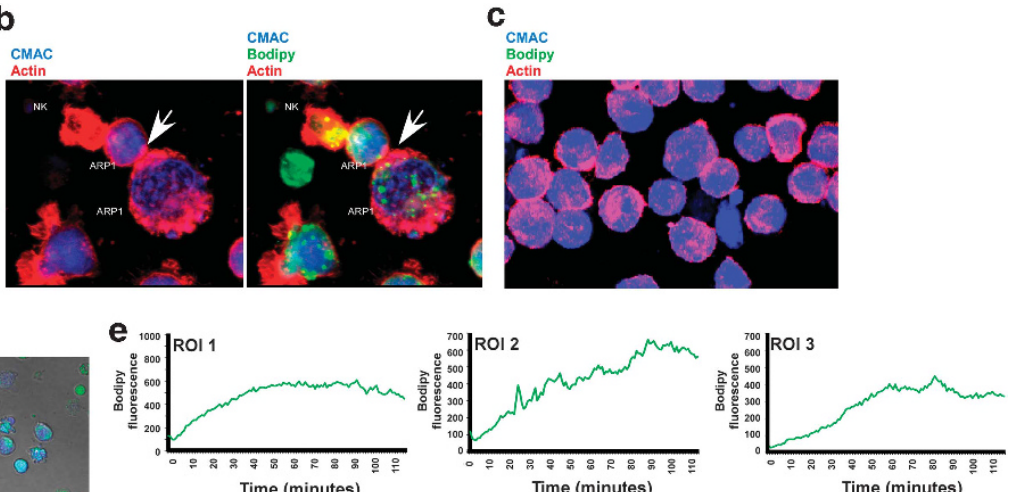

f
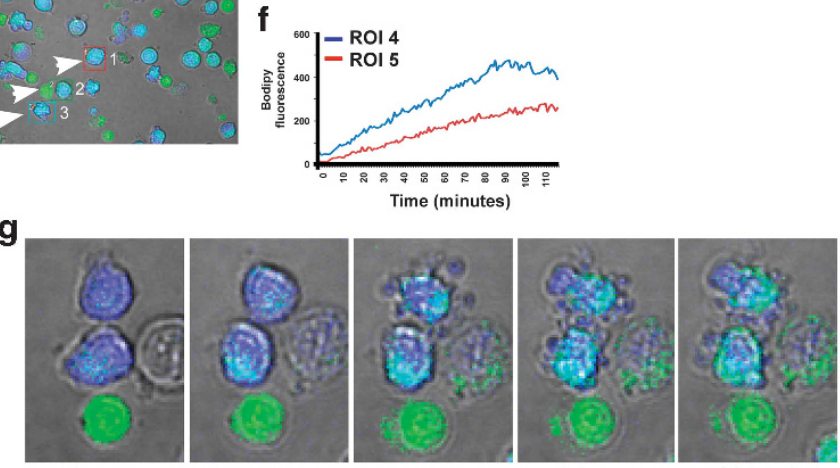

$90 \mathrm{~min}$

$120 \mathrm{~min}$

Figure 2 CB-NK contents are transferred to ARP1 cells via lipid vesicles or lipid droplets (LDs). (a) CB-NK and ARP1 cells were co-cultured in cell pellet for 10 min. The two images are the same showing ARP1 cells stained in blue (CMAC) and CB-NK in green (bodipy). Actin is shown in red. CMAC and actin are shown in the first image and the three colors together in the second image. Arrows indicate transfer of CB-NK content in green into ARP1 cells, indicating lipid trafficking. (b) Same experiment as panel a showing one CB-NK transferring content to one ARP1 cell and this ARP1 cell transferring content to a secondary ARP1 cell through the immunological synapse. (c) ARP1 cells alone as control stained with CMAC and actin in red. (d) Different time points taken from a time lapse in vivo imaging for $2 \mathrm{~h}$ (Supplementary Movie S1) where ARP1 cells were stained in blue (CMAC) and CB-NK in green (bodipy). Five different ARP1 cells are indicated in regions of interest (ROIs). ROls 1-3 indicate three ARP1 cells that had been touched by the same CB-NK. ROI 4 indicates one ARP1 cell touched by a CB-NK and then this ARP1 cell transfers the CB-NK content into another ARP1 cell (ROI 5). (e) Analysis of the increase during time of green staining (bodipy) in ROls 1-3 from panel d. (f) Analysis of the increase during time of green staining (bodipy) in ROls 4 and 5 in panel d. (g) Amplified images from ROls 4 and 5 in panel $\mathbf{d}$ to show ARP1 cells showing membrane blebbing. See also Supplementary Movie S1

GrB- and Caspase-3-independent CB-NK cytotoxicity was also confirmed for other MM cell lines (Supplementary Figure S3C). However, cytotoxicity of PB-NK from healthy individuals versus K562 and ARP1 was GrB and Caspase-3 dependent (Supplementary Figure S4B), providing another unique characteristic of CB-NK. Because CB-NK degranulation indicates release of lysosomes, we measured lysosomes in K562 and ARP1 cells after CB-NK. As controls, CD19 ${ }^{+}$ cells from healthy individuals were used. Whereas in $\mathrm{K} 562$ and $\mathrm{CD} 19^{+}$cells there was an increase in lysosomes after CB-NK (Figure 5f, $P<0.0001$ ), in ARP1 there was a decrease (Figure 5f, $P<0.0001$ ), confirming that different mechanisms of CB-NK cytotoxicity against MM cells exist.

CB-NK induce lysosomal cell death in MM cells, and NKG2D and NKP30 are involved in this mechanism. We aimed to understand the mechanisms underlying CB-NK cytotoxicity versus MM cells. We had observed lipid trafficking between cells, cytotoxicity that was independent GrB and Caspase-3 and decreased lysosomes after CB-NK exposure (Figure 5). Granulysin can cause Caspase-3independent cell death with ER stress and lysosomal permeabilization. ${ }^{7,8}$ After permeabilization, lysosomes release cathepsins leading to a Caspase-3-independent cell killing called 'Iysosomal cell death'. ${ }^{8}$ We hypothesized that CB-NK induce lysosomal cell death in MM cells. We analyzed lysosome markers (Lyso-tracker and Rab7, which also defines lysosomes) and the impact of cysteine cathepsins in CB-NK cytotoxicity. After 40 min with CB-NK, ARP1 cells had reduced lysosomes levels (Figures $6 a, b$, and $\mathrm{d}, P<0.0001)$. Further, cathepsins inhibition reduced CB-NK cytotoxicity versus ARP1 and not versus K562 (Figure 6e), confirming a lysosomal cell death for MM cells; which was confirmed in other MM cell lines, by detecting both a decrease in Rab7 and the impact of cathepsins in CB-NK cytotoxicity (Supplementary Figures S3D and E). Primary MM CD138 ${ }^{+}$cells also showed decreased lysosomes levels after CB-NK (Supplementary Figure S3F). Interestingly, cytotoxicity of PB-NK from healthy individuals versus K562 or ARP1 cells was not affected by cathepsins inhibition (Supplementary Figure S4C), indicating another unique feature of CB-NK.

ROS were also decreased in ARP1 cells (Figures $6 \mathrm{c}$ and $\mathrm{f}$, $P<0.0001)$ and in primary MM CD138 ${ }^{+}$cells after CB-NK exposure (Supplementary Figure S3F). As GrB leads to ROS production $^{4}$ and Caspase-3-dependent cell death, we 
a

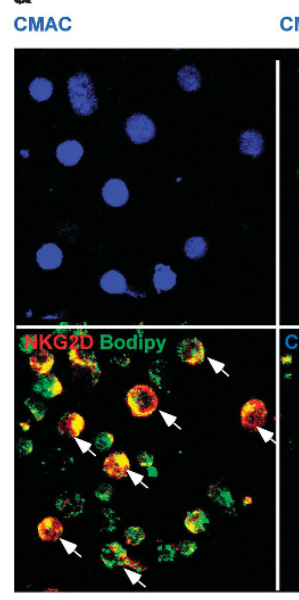

C

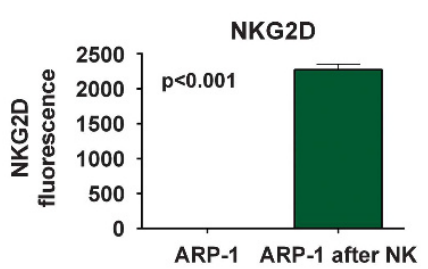

CMAC Bodipy

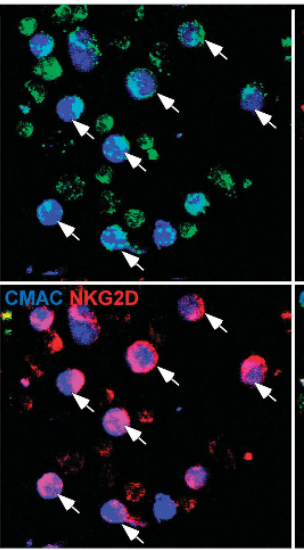

$$
\text { K }
$$

NKG2D

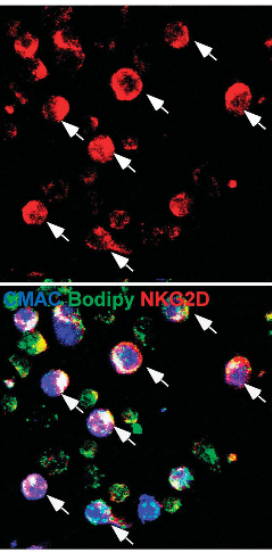

NKP30

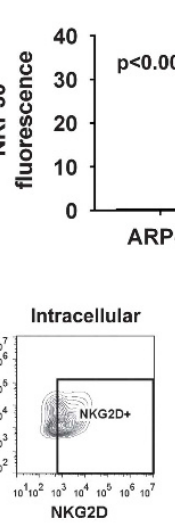

ARP-1 ARP-1 after NK

f
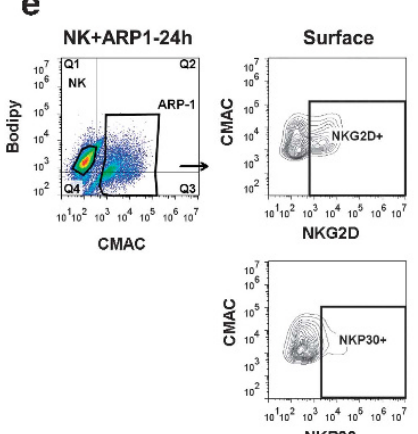

NKP30

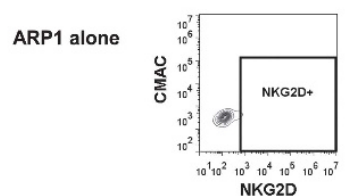

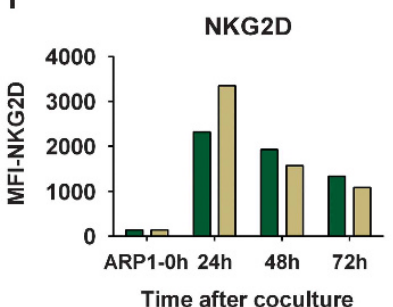

Time after coculture b

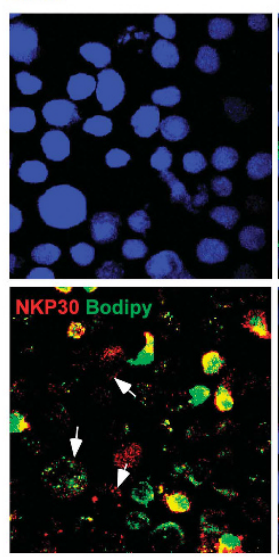

CMAC Bodipy

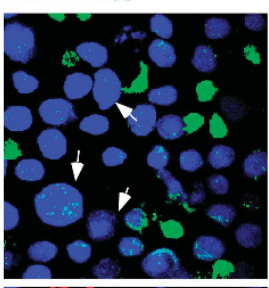

NKP30

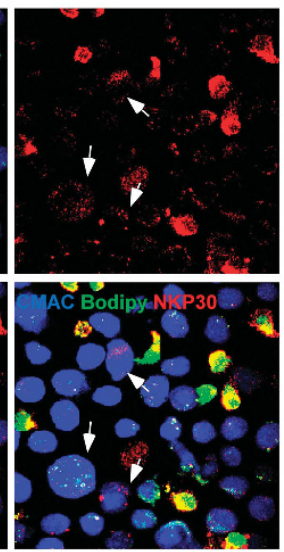

d
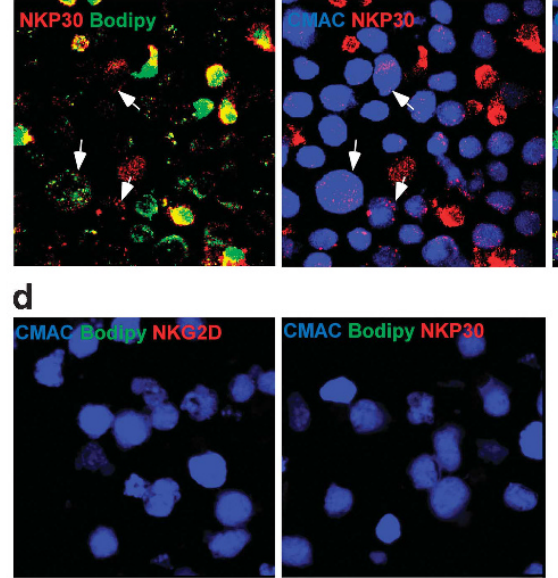
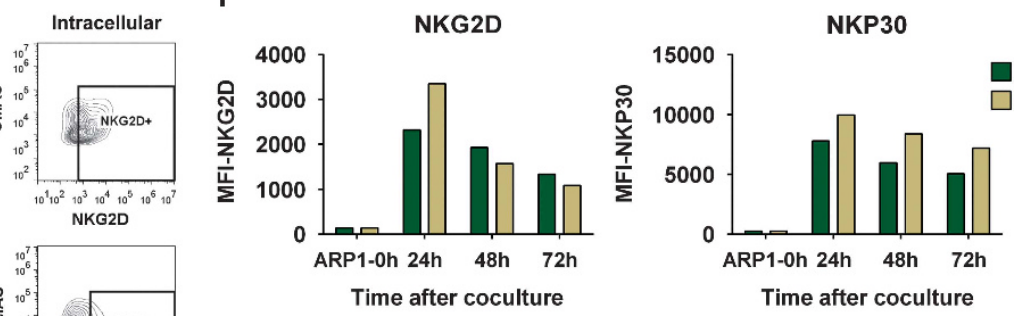

Surface

$\square$ Intracellular

Figure 3 NKG2D and NKP30 are transferred into MM cells: (a) NKG2D and (b) NKP30 in ARP1 cells after co-culturing with CB-NK for 20 min. ARP1 cells were stained in blue (CMAC) and CB-NK in green (bodipy). NKG2D and NKP30 are indicated in red. All the images represent the same showing different combinations of the three colors. Arrows indicate ARP1 cells with NKG2D or NKP30. (c) Fluorescence values obtained from images in panels a and $\mathbf{b}$. Bars represent mean \pm S.E.M. (d) As control, ARP1 cells alone were stained for NKG2D and NKP30 with the same settings than in images $\mathbf{a}$ and $\mathbf{b}$. Results from panels $\mathbf{a}$ to $\mathbf{d}$ were confirmed in three different experiments. (e and f) Detection by flow cytometry of NKG2D and NKP30 in ARP1 cells after 24, 48 and $72 \mathrm{~h}$ of co-culture with CB-NK. ARP1 cells were gated based on CMAC staining. (e) Representative plot of the experiment at $24 \mathrm{~h}$ of co-culture. ARP1 cells unstained alone were used as control. (f) Median fluorescence intensity (MFI) values for NKG2D and NKP30 in ARP1 cells at the different times of co-culture, performing both surface and intracellular staining

investigated whether this pathway was responsible for the decreased ROS levels observed. Inhibition of GrB and Caspase-3 did not abrogate the decrease in ROS (Figure 6f), confirming that GrB and Caspase-3 in MM cells neither activate cell death nor ROS production. We investigated whether the decreased ROS after CB-NK treatment was only characteristic of MM cells. In other tumor cells analyzed (K562), there was also a decrease in ROS after CBNK treatment (Figure 6g, $P<0.0001$ ); however, CD19 ${ }^{+}$cells from healthy individuals showed an increase in ROS after CB-NK (Figure 6g, $P<0.0001$ ).

To determine whether NKG2D and NKP30 were involved in the decreased levels of lysosomes and ROS in MM cells after CB-NK exposure, these receptors were blocked, and lysosomes and ROS levels analyzed. Blocking either NKG2D (Figure 6h, $P<0.0001$ ) or NKP30 (Figure 6i, $P<0.0001$ ) abrogated the CB-NK effect on the decreased lysosome and ROS levels in MM cells after CB-NK exposure, indicating that 

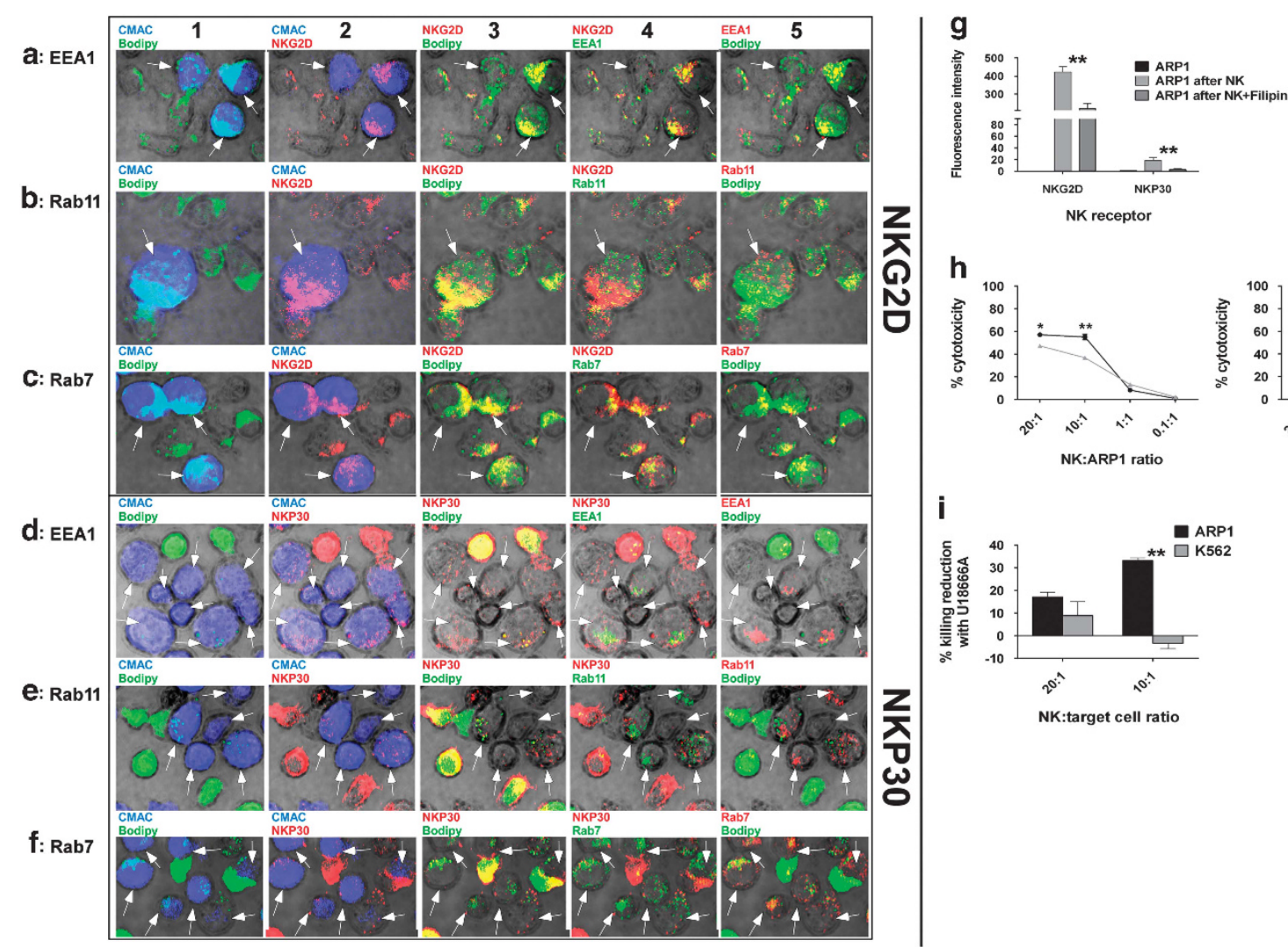

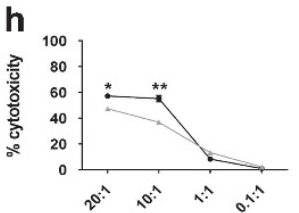

NK:ARP1 ratio

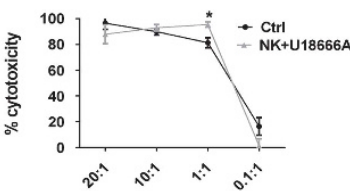

i

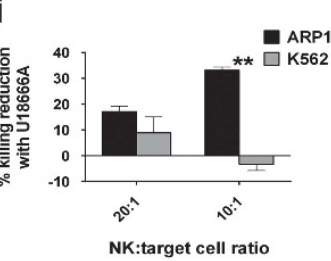

Figure 4 The transfer of NKG2D and NKP30 into MM cells involves lipid trafficking and the endocytic pathway: Co-localization of NKG2D with (a) EEA1, (b) Rab11 and (c) Rab7 and of NKP30 with (d) EEA1, (e) Rab11 and (f) Rab7 in ARP1 cells after being co-cultured with CB-NK for 20 min. ARP1 cells were stained in blue (CMAC) and CB-NK in green (bodipy), NKG2D and NKP30 are indicated in red. All the images in one row are the same but showing different combinations of colors. In column 1, CMAC and bodipy are shown to indicate ARP1 cells and CB-NK. In column 2, NKG2D or NKP30 and CMAC are shown indicating ARP1 cells with NKG2D or NKP30. In column 3, bodipy in green and NKG2D and NKP30 in red indicate co-localization (yellow) of NKG2D and NKP30 with lipids. In column 4, NKG2D and NKP30 are indicated in red and the endocytic marker in green. In column 5, bodipy in green and the endocytic marker in red indicate co-localization of lipid vesicles with endocytic pathway. Arrows indicate ARP1 cells. (g) NKG2D and NKP30 expression in ARP1 cells alone (ARP1 alone), after being co-cultured with CB-NK for 40 min (ARP1 after NK) and after being co-cultured for 40 min with CB-NK previously incubated with Filipin III (ARP1 after NK + Filipin). (h) CB-NK cytotoxicity reduction versus ARP1 and K562 cells after incubating previously CB-NK with U18666A for $2 \mathrm{~h}$. (i) CB-NK cytotoxicity reduction of results shown in panel h. Bars represent mean \pm S.E.M. ${ }^{\star} P \leq 0.05^{\star}$. ${ }^{\star \star} P \leq 0.0001$

both receptors were involved in the cell death mediated by CB-NK.

MM stroma decreases CB-NK cytotoxicity. Because of the protective role of the stroma in $\mathrm{MM}$, we analyzed the impact of MM stroma in CB-NK cytotoxicity. MM stroma reduced CB-NK cytotoxicity for MM cells (ARP1, RPMI and KMM1, Figure 7a, $P<0.05)$. We observed that both MM cells and CB-NK adhered strongly to the stroma making difficult the formation of the immunological synapse between CB-NK and MM cells. However, we still observed vesicle transfer from CB-NK to $\mathrm{MM}$ cells and also secondary transfer between MM cells (Figure $7 \mathrm{~b}$ ). Because CXCL12 tethers hemopoietic cells into the stroma, ${ }^{28}$ we measured CXCL12 secretion by the stroma and observed a reduced $\mathrm{CXCL} 12$ secretion after the addition of $\mathrm{MM}$ cells or MM cells and CB-NK (Figure 7c, $P<0.05$ ), suggesting a possible role for CXCL12 in reducing the anti-MM cytotoxicity of CB-NK. Therefore we performed cytotoxicity assay adding AMD3100, which inhibits binding of CXCL12 to CXCR4 in hemopoietic cells. A slight recovery in CB-NK cytotoxicity versus RPMI MM cell line was observed
( $P=0.09$, Figure $7 \mathrm{~d})$, providing evidence to support this possibility.

CB-NK cytotoxicity is transmissible between MM cells. We demonstrated lipid-protein trafficking between MM cells after CB-NK contact. Recipient MM cells appeared to become apoptotic with membrane blebbing (Figure $2 \mathrm{~g}$ and Supplementary Movie S1). We examined whether NKG2D and NKP30 were secondarily transferred from ARP1 cells previously exposed to CB-NK ('primary $\left(1^{\circ}\right)$ ARP1 cells') to adjacent ARP1 cells ('secondary ( $\left.2^{\circ}\right)$ ARP1 cells'). Bodipylabeled CB-NK and CMAC-labeled ARP1 cells were cocultured for $40 \mathrm{~min}$; these ARP1 cells were defined as $1^{\circ}$ ARP 1 cells. Alive $1^{\circ}$ ARP 1 cells were then FACS-sorted. These sorted $1^{\circ}$ ARP1 cells were then either left alone $\left(1^{\circ}\right.$ ARP1 alone) or co-cultured $\left(1^{\circ}\right.$ ARP 1 with $2^{\circ}$ ARP 1$)$ in cell pellet for another $40 \mathrm{~min}$ with unstained fresh $2^{\circ}$ ARP1 ( $2^{\circ}$ ARP1 after $1^{\circ}$ ARP1) cells allowing differentiation between the two ARP1 cell populations $\left(\mathrm{CMAC}^{+} 1^{\circ} \mathrm{ARP} 1\right.$ cells versus unstained $2^{\circ}$ ARP1 cells). Then, in these two sets of ARP1 cells, we analyzed the expression of NKG2D and NKP30 (Figure 8a). 
a
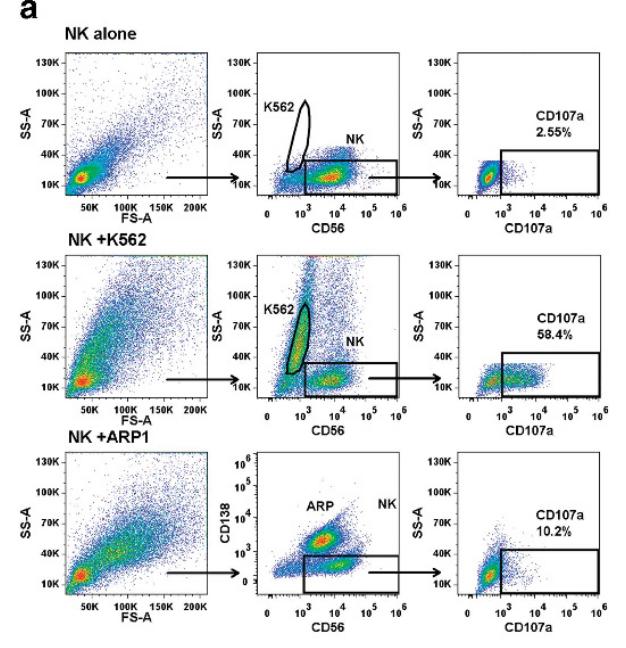

b

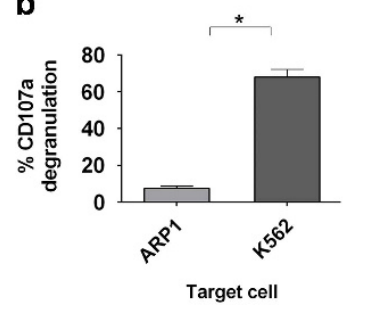

d

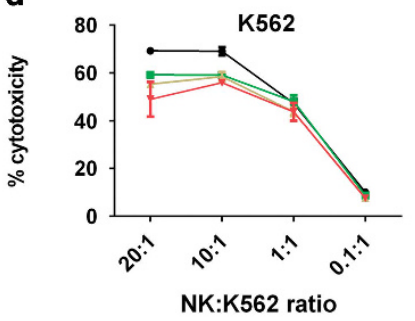

e

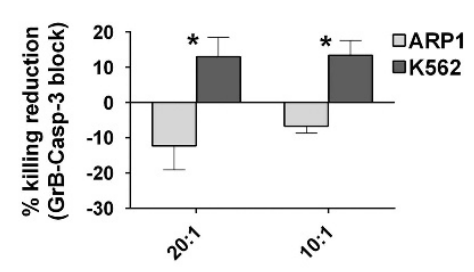

NK:target cell ratio

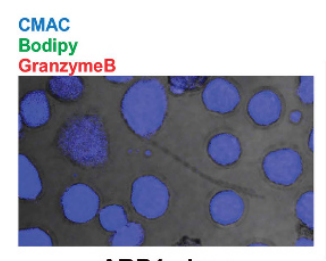

ARP1 alone

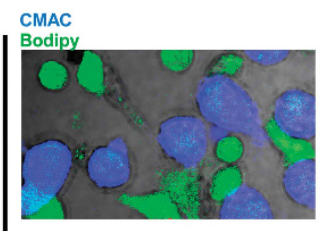

$4+2-10$

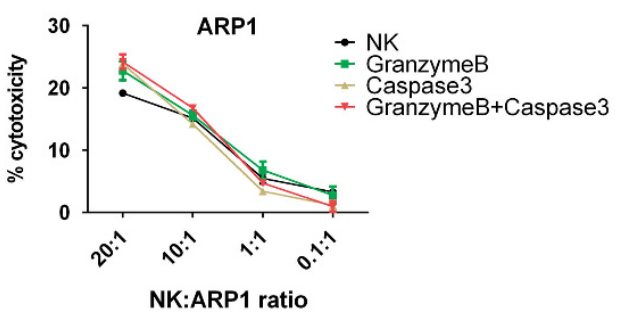

f

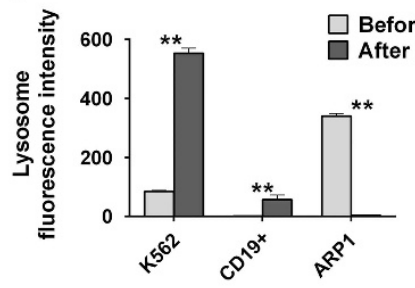

Figure 5 The mechanisms underlying CB-NK-mediated cytotoxicity differ between target cells: (a) Degranulation (CD107a expression) of CB-NK after $4 \mathrm{~h}$ of incubation with K562 or ARP1 cells. (b) Mean \pm S.E.M. values from a representative experiment shown in a $(n=4)$. (c) GrB expression in ARP1 cells after being co-cultured with CB-NK for $20 \mathrm{~min}$. ARP1 cells were stained in blue (CMAC) and CB-NK in green (bodipy). GrB is indicated in red. (d) CB-NK cytotoxicity reduction after inhibiting GrB and Caspase-3. Killing is shown for control CB-NK, after inhibiting GrB in CB-NK, Caspase-3 in target cells and both, GrB in CB-NK and Caspase-3 in target cells. (e) Percentage of killing reduction after inhibiting $\mathrm{GrB}$ and Capase-3 in four different $\mathrm{CB}$ units of the representative experiment shown in $\mathrm{d}$. (f) Lysosome levels before and after $\mathrm{CB}-\mathrm{NK}$ exposure for $40 \mathrm{~min}$ for $\mathrm{K} 562$ cells, $\mathrm{CD} 19^{+}$cells from a healthy donor and ARP1 cells. Bars represent mean \pm S.E.M. ${ }^{\star} P \leq 0.05 .{ }^{\star \star} P \leq 0.0001$

NKG2D (Figures $8 \mathrm{~b}$ and $\mathrm{d}, P<0.0001$ ) and NKP30 (Figures $8 \mathrm{c}$ and $\mathrm{e}, P<0.0001)$ were transferred secondarily to neighboring $2^{\circ}$ ARP1 cells ( $2^{\circ}$ ARP1 after $1^{\circ}$ ARP1 in Figure 8) from $1^{\circ}$ ARP1 cells, which had primarily synapsed with NK cells ( $1^{\circ}$ ARP1 with $2^{\circ}$ ARP1 in Figure 8). Co-staining for actin indicated that this transfer occurred through an immunological synapse between ARP1 cells, especially for NKG2D (Figures 8d and e).

As NKG2D and NKP30 were transferred to $2^{\circ}$ ARP1 cells, we also analyzed the downstream effects of this transfer on lysosomes and ROS levels and whether this effect was translated in some indirect cytotoxicity of CB-NK against these $2^{\circ}$ ARP1 cells. These $2^{\circ}$ ARP1 cells demonstrated decreased lysosome and ROS levels after contact with $1^{\circ}$ ARP1 cells $\left(2^{\circ}\right.$ ARP1 after $1^{\circ}$ ARP1: in Figures $8 f$ and $g$, $P<0.0001$ ), in comparison to the control sorted ARP1 cells (ARP1 ctrl: in Figures $8 f$ and g). We also noticed a compensating effect between the two populations of ARP1 cells, as after $1^{\circ}$ ARP 1 cells were exposed to CB-NK and then incubated with $2^{\circ}$ ARP1 cells, the $1^{\circ}$ ARP 1 cells began to normalize their loss in lysosomes and ROS $\left(1^{\circ}\right.$ ARP1 alone versus $1^{\circ}$ ARP1 with $2^{\circ}$ ARP1: in Figures $8 f$ and $g$, $P<0.0001$ ).

To determine whether CB-NK cytotoxicity was also transmitted between ARP1 cells, we performed cytotoxicity assay by adding $1^{\circ}$ ARP1-sorted cells as effectors and $2^{\circ}$ ARP 1 cells as targets. $1^{\circ}$ ARP 1 cells, which previously synapsed with CB-NK, demonstrated $7 \%$ cytotoxicity against $2^{\circ}$ ARP1 cells at a 40:1 effector: target ratio. Although this cytotoxicity decreased at lower effector:target cell ratios, it was greater than the cytotoxicity of the control ARP1 cells untouched by CB-NK (Figure 8h), indicating a transmissible cell death from CB-NK to MM cells and then from these MM cells to neighboring MM cells. Finally, we tested whether the MM stroma impacted on this indirect 'domino-effect' cytotoxicity. Cytotoxicity assay containing MM stroma reduced indirect cytotoxicity of $1^{\circ}$ ARP1 versus $2^{\circ}$ ARP1 at 20:1 effector:target cell ratio (Figure 8i), suggesting that $\mathrm{MM}$ stroma may reduce 'domino-effect' cytotoxicity.

\section{Discussion}

We show a novel CB-NK-mediated cytotoxicity against MM cells that is GrB and Caspase-3 independent, involves transmissible cytotoxicity between CB-NK and MM cells via lipid-protein vesicle trafficking and secondarily transferred from these MM cells to neighboring MM cells. This lipidprotein transfer involves NKG2D and NKP30 receptors, causes lysosomal cell death and reduced ROS levels. This cytotoxicity appears to be unique of CB-NK and different from 


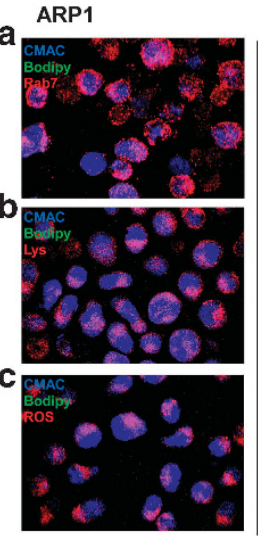

NK+ARP1
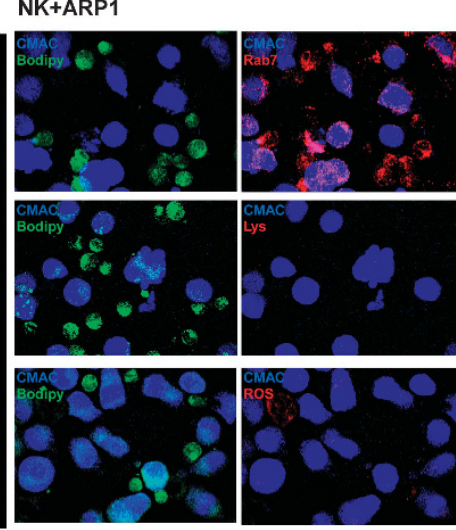

d
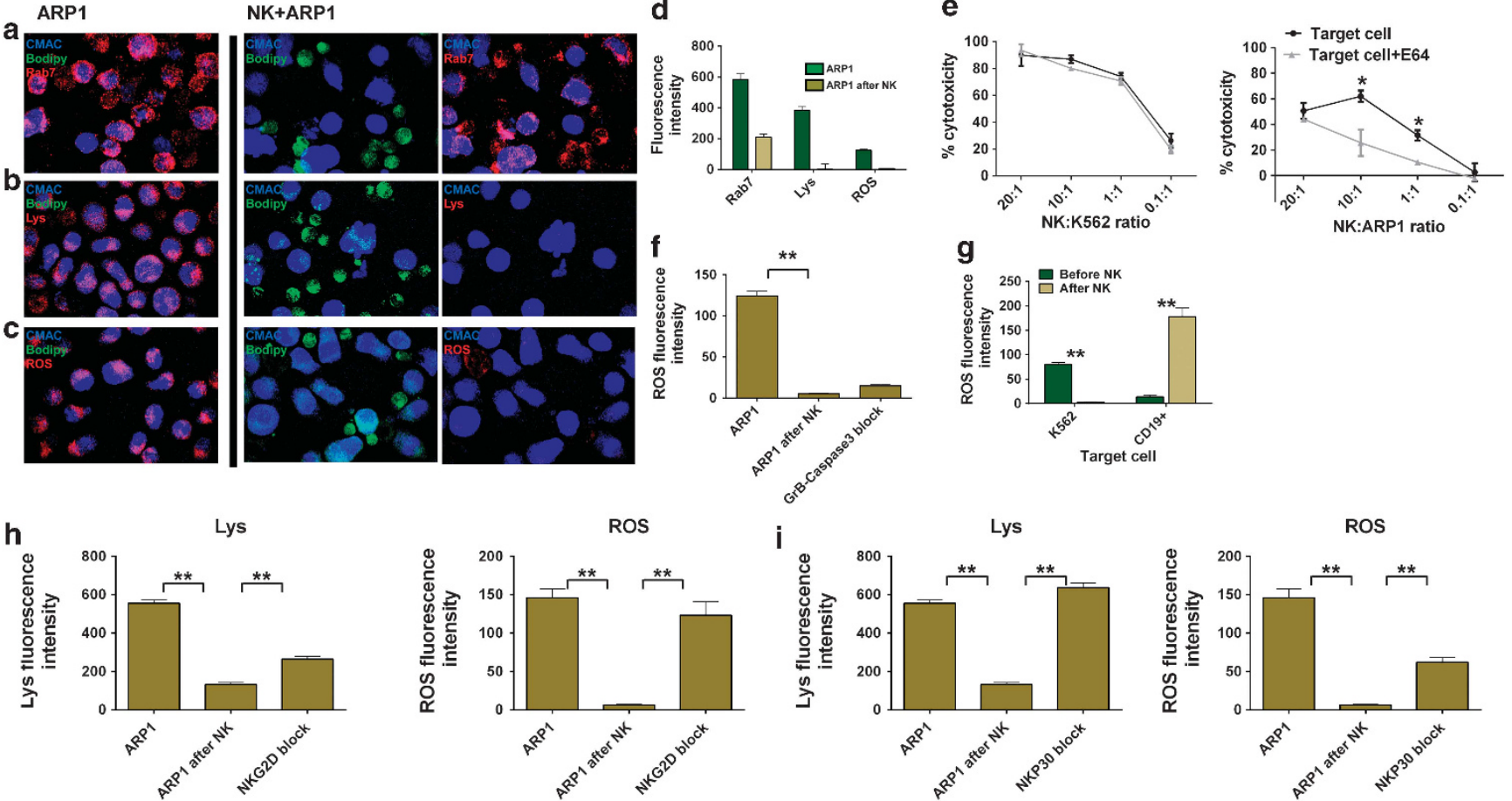

Figure 6 CB-NK induce lysosomal cell death in MM cells, and NKG2D and NKP30 are involved in this mechanism: (a-d) Analysis of lysosomes (Rab7 and Lyso-trracker: Lys) and ROS production in ARP1 cells before and after co-culture in cell pellet with CB-NK for 40 min. ARP1 cells were labeled in blue (CMAC), CB-NK in green (bodipy) and each marker is indicated in red. (a) Late endocytic and lysosome marker Rab7. (b) Lysosome marker (Lyso-tracker). (c) ROS production. (d) Mean \pm S.E.M. values of slides analyzed from a to $\mathbf{d}$. Results from a to $\mathbf{c}$ were confirmed in three different experiments. (e) CB-NK cytotoxicity reduction after incubating target cells (K562 and ARP1) with E64 (a cysteine cathepsin inhibitor). (f) ROS variation after inhibiting GrB in CB-NK and Caspase-3 in ARP1 cells and co-culturing for 40 min. (g) ROS levels before and after CBNK exposure for $40 \mathrm{~min}$ for K562 cells and CD19 ${ }^{+}$cells from healthy individuals. (h and i) Variation of lysosome (Lys) and ROS levels after CB-NK exposure for 40 min (ARP1 after NK) and after blocking (h) NKG2D and (i) NKP30 in CB-NK (NKG2D block and NKP30 block). Lysosomes were determined with the Lyso-tracker. Bars represent mean \pm S.E.M. ${ }^{* *} P \leq 0.0001$

that seen against K562 cells where NKG2D and NKP30 do not have such a relevant role.

Lysosomal cell death happens after ruptured lysosomes release cathepsins leading to Caspase-3-independent cell death, which can show membrane blebbing, and which, depending on the magnitude of Iysosomal permeabilization, will activate an apoptotic-like programmed cell death or necrosis cell death. ${ }^{29}$ Lipids and Granulysin are involved in this type of cell death as lipids destabilize lysosomes ${ }^{30}$ and Granulysin mediates a Caspase-3-independent lysosomal cell death with membrane blebbing. ${ }^{8}$ These studies support our findings where we show a transmissible MM cell death mediated by CB-NK, involving lipid-protein transfer with a decrease in lysosomes, cell death dependent on Cathepsins and showing membrane blebbing in MM cells. NKG2D and NKP30, associated with the decreased lysosomes levels, were also indirectly transferred to new $\mathrm{MM}$ cells indicating their possible role in this indirect cytotoxicity. Although no measurement of Granulysin was made, our findings do not discard a possible role of Granulysin in this lysosomal cell death.

Cells exchange information via exosomes/lipid vesicles, and NK release cytotoxic exosomes/vesicles expressing NK-activating receptors. ${ }^{3,31}$ Lipids inhibit autophagy ${ }^{32}$ and can activate ER stress-associated cell death. ${ }^{33}$ Here we show lipid trafficking between cells and that this exchange of 'information' in lipid structures, which contain NKG2D and NKP30, occurs between CB-NK and MM cells and afterwards between adjacent $\mathrm{MM}$ cells being associated with cytotoxicity against MM cells. Even the bone marrow stroma reduced this CB-NK direct and indirect cytotoxicity, it was not an impediment for this lipid transfer from CB-NK to MM cells and secondarily to neighboring MM cells.

GrB-induced NK death is usually accompanied by increased ROS production, a process which is Caspase-3 dependent. ${ }^{4}$ We found that even though GrB is transferred into ARP1 cells by CB-NK, the GrB and Caspase-3 pathway was not involved neither in the killing of ARP1 cells nor in the decreased ROS levels detected. By comparison, NKG2Dand NKP30-associated MM cell killing was associated with decreased ROS, providing evidence for a role for these receptors. As ROS is associated with survival in tumor cells and with a lowering of the interaction between cancer cells and the immune system, ${ }^{34,35}$ this CB-NK-associated ROS reduction might make tumor cells more vulnerable to cellbased immunotherapeutic strategies.

MM cells, which are susceptible to ER stress cell death, have high autophagy levels. ${ }^{18,36}$ Autophagy uses lysosomes for degradation, ${ }^{37}$ associates to increased $\mathrm{ROS}^{38}$ and contributes to chemotherapeutic resistance. ${ }^{17}$ We observed decreased lysosomes and ROS in MM cells after CB-NK 


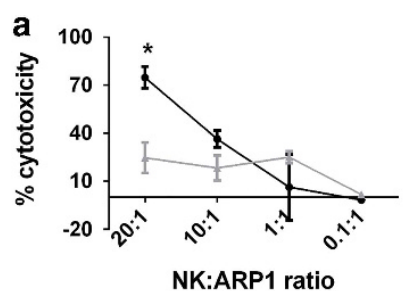

NK:ARP1 ratio
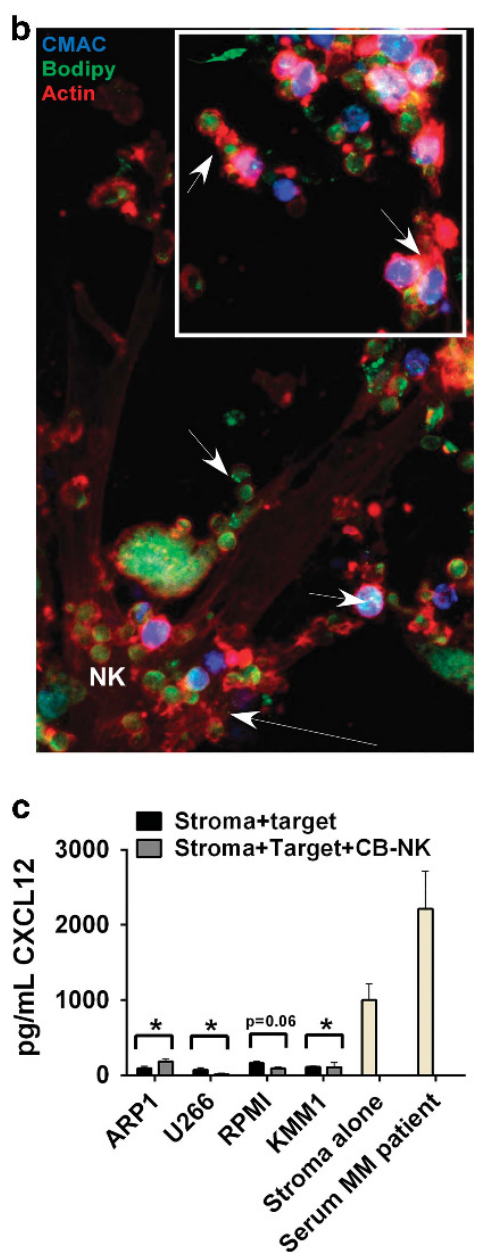

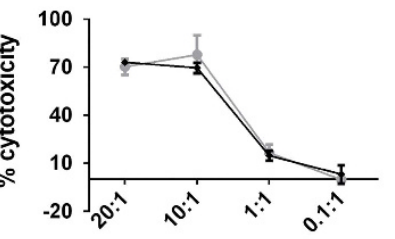

NK:U266 ratio

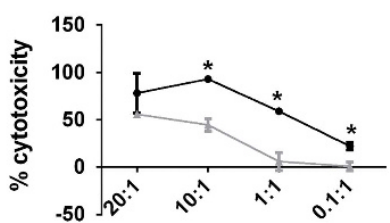

NK:RPMI ratio

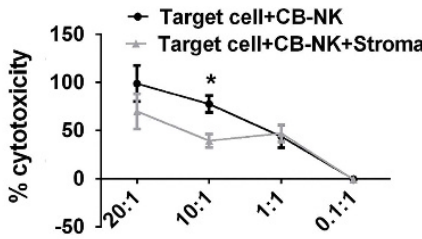

NK:KMM1 ratio
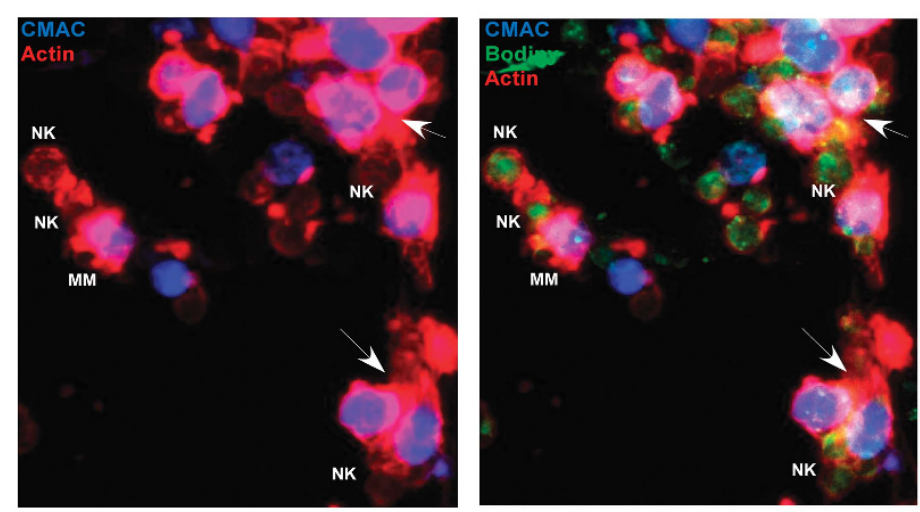

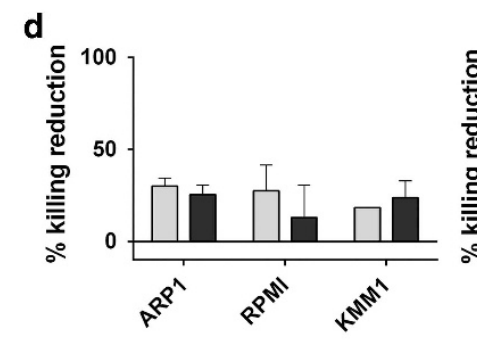

20:1 ratio

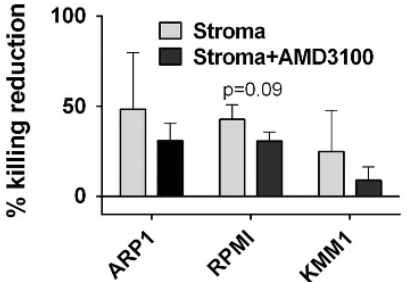

10:1 ratio

Figure 7 MM stroma decreases CB-NK cytotoxicity: (a) CB-NK cytotoxicity reduction after adding MM stromal cells into cytotoxicity assays. Cytotoxicity assays for MM cells were done in parallel either adding (Target cell $+\mathrm{CB}+\mathrm{NK}+$ Stroma) or not adding (Target cell + CB-NK) MM stromal cells to the assay. (b) Confocal fluorescence image of stromal MM cells with CB-NK (in green with bodipy) and KMM1 MM cells (in blue with CMAC). Actin is shown in red. Stromal cells can be visualized by the actin filaments. Arrows in the lower part show CB-NK adhered to stromal cells. Arrows in the upper part show transfer of CB-NK content to MM cells and also secondarily between MM cells. The white square is shown amplified in the right side showing either two colors (CMAC and actin) or the three colors together (CMAC, bodipy and actin). Same images were confirmed for other MM cell lines. (c) CXCL12 levels in the supernatant of cytotoxicity assays with MM cells adding either stroma with target MM cells and CB-NK (Stroma + target + CB-NK) or stroma with target cells alone (Stroma + target). Serum MM patient was added as positive control. $P$-values represent analysis of stroma alone versus stroma + target. (d) Cytotoxicity CB-NK reduction at 20:1 or 10:1 effector:target ratio, after adding (Stroma + AMD3100) or not adding (Stroma) AMD3100 to the cytotoxicity assay with stroma, CB-NK and MM cells. ${ }^{*} P \leq 0.05$

exposure, which could suggest decreased autophagy levels. Although an extensive study of autophagy markers was not undertaken here, the possible autophagy reduction in $\mathrm{MM}$ cells by CB-NK could reduce the refractoriness, which happens in some MM patients after $\mathrm{PI}$ treatment. ${ }^{17,19}$

In summary, we show a unique mechanism of CB-NK cytotoxicity that involves transmissible cytotoxicity between cells, where lipids appear as 'vehicles' to perform this mechanism. This opens new pathways for the use of CB-NK as a cellular immunotherapy option and for developing therapeutic strategies using CB-NK-derived lipids.

\section{Material and Methods}

Ethics statement. All research involving human materials was approved by the MD Anderson Cancer Center (MDACC) Institutional Review Board (IRB). CB units were obtained from healthy donors who gave written informed consent. All animal work was performed under an MDACC Institutional Animal Care and Use Committee (IACUC)-approved protocol specific to this study. 
a co-culture for $\mathbf{4 0}$ minutes
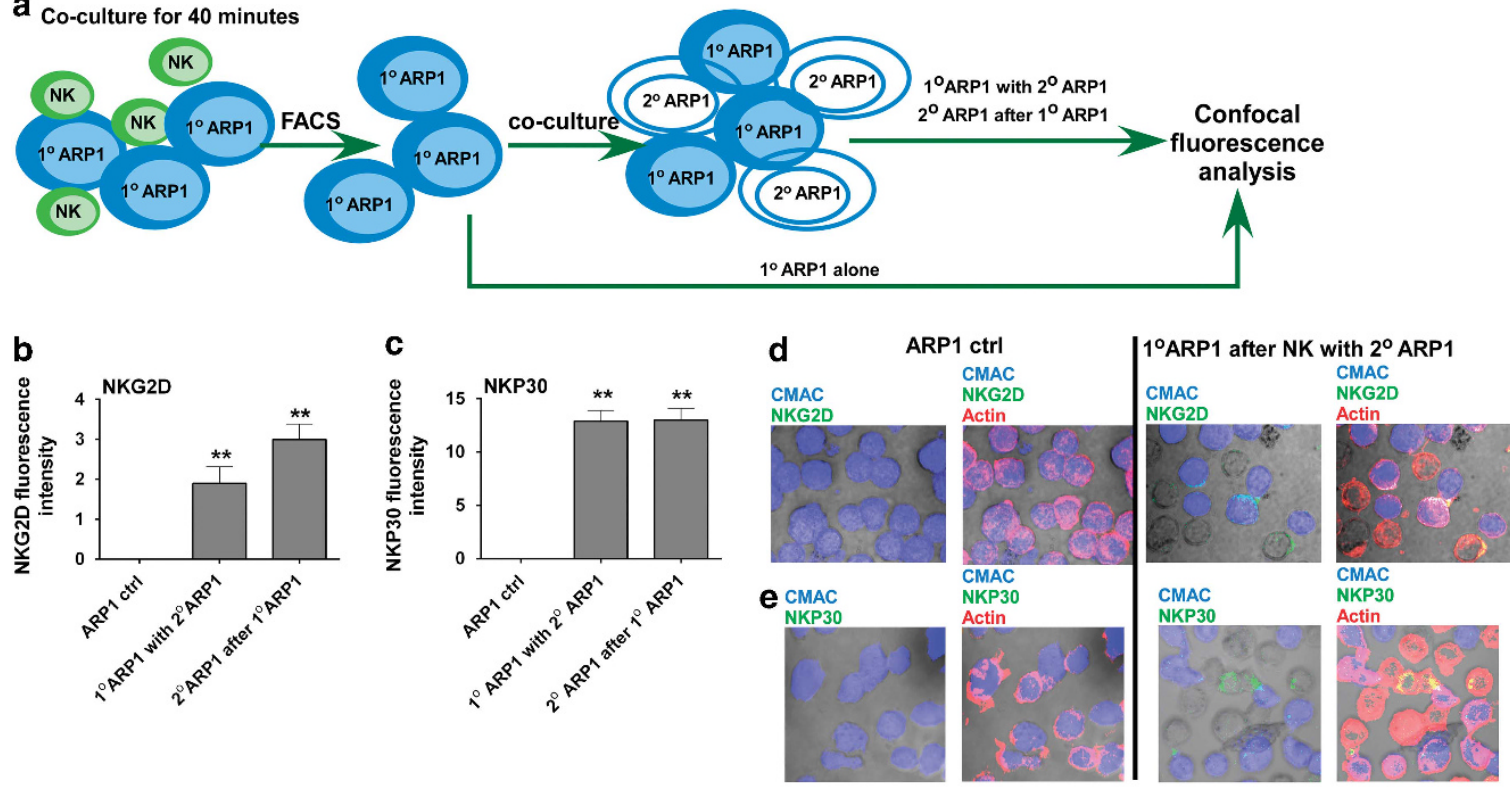

f

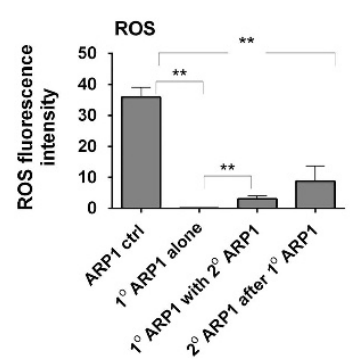

g

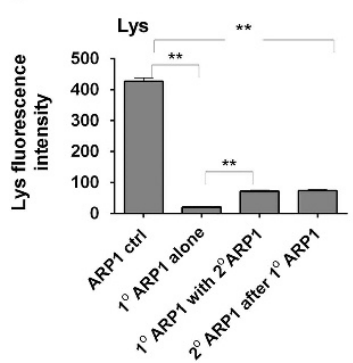

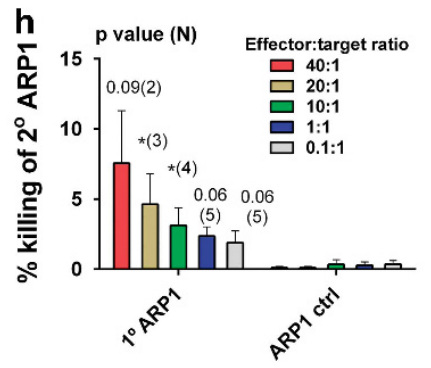

Effector cell

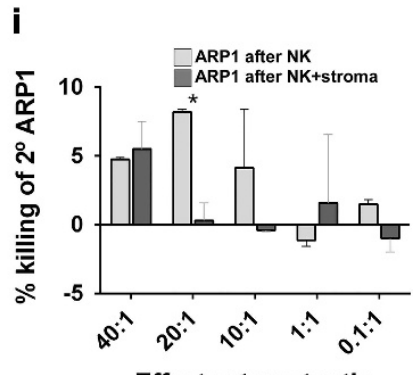

Effector:target ratio

Figure 8 CB-NK cytotoxicity is transmissible between MM cells: (a) Design experiment for results from $\mathbf{b}$ to $\mathbf{h}$. Bodipy-labeled CB-NK and CMAC-labeled ARP1 cells $\left(1^{\circ} \mathrm{ARP} 1\right.$ cells) were co-cultured in cell pellet for $40 \mathrm{~min}$, then $1^{\circ} \mathrm{ARP} 1$ cells were FACS sorted and half of them were left alone $\left(1^{\circ} \mathrm{ARP} 1\right.$ alone), and half of them $\left(1^{\circ} \mathrm{ARP} 1\right.$ with $2^{\circ}$ ARP1) were co-cultured in cell pellet for 40 min with fresh unstained ARP1 cells ( $2^{\circ}$ ARP1 cells after $1^{\circ}$ ARP1), then confocal fluorescence experiments and analyses were performed discriminating between the two sets of ARP1 cells based on $1^{\circ}$ ARP1 cells labeled in blue (CMAC) and $2^{\circ}$ ARP1 cells unstained. (b and $\left.\mathbf{c}\right)$ NKG2D (b) and NKP30 (c) expression in $1^{\circ}$ ARP1 cells and $2^{\circ}$ ARP1 cells. Bars represent mean \pm S.E.M. (d and e) Representative images of NKG2D (d) and NKP30 (e) expression in green in both sets of ARP1 cells of values obtained in $\mathbf{b}$ and $\mathbf{c}$. Actin is shown in red. Lysosome (f) and ROS $(\mathbf{g})$ expression in the two sets of ARP1 cells. ARP1 ctrl: indicates ARP1 cells alone sorted as control for the experiments. Bars represent mean \pm S.E.M. Results from $\mathbf{b}$ to $\mathbf{g}$ were confirmed in three different experiments. (h) Cytotoxicity assay performed adding as effector cells $1^{\circ}$ ARP1 sorted cells and as targets fresh ARP1 cells. Effectors were added at different effector:target cell ratios. In parallel, ARP1 cells alone were sorted and added as effectors to the cytotoxicity assay as control. (i) Cytotoxicity reduction of $1^{\circ}$ ARP1 (as effectors) versus fresh ARP1 cells (as targets) after adding (ARP1 after NK + stroma) or not adding (ARP1 after NK) stroma to the assay. ${ }^{*} P \leq 0.05 .{ }^{*} P \leq 0.0001$

Cell cultures. K562-based antigen-presenting cells expressing membranebound IL-21 ('Clone 9.mblL21') was kindly provided by Dean A Lee and Laurence JN Cooper (MDACC, Houston, TX, USA). CB-NK were obtained using methods as previously described. ${ }^{20}$ Briefly, NK cells were MACS-selected (Miltenyi Biotec, San Diego, CA, USA) from $\mathrm{CB}$ mononuclear cells (CBMC) and co-cultured with irradiated Clone 9.mblL21 cells at a ratio of 2:1 (Clone 9: NK cells). IL-2 (Proleukin; Chiron, Emeryville, CA, USA) was added at $400 \mathrm{UI} / \mathrm{ml}$ every other day. At day 14, CB-NK were harvested for all the experiments. Culture media was comprised of $45 \%$ RPMI- 1640 (Cellgro, Manassas, VA, USA) and 45\% Click's media (Irvine Scientific, Santa Ana, CA, USA) supplemented with 10\% AB human serum (Atlanta Biologicals, Lawrenceville, GA, USA). ARP1 cell line was kindly provided by Multiple Myeloma Research Center (Little Rock, AK, USA) to Lymphoma/Myeloma Department (M.D. Anderson Cancer Center, Houston, TX, USA). K562, U266, RPMI and KMM1 cell lines were obtained from American Type Culture Collection (ATCC, Rockville, MD, USA). K562, ARP1 and KMM1 were cultured in RPMI supplemented with $10 \%$ fetal bovine serum (FBS) and U266 with $15 \%$ FBS. CD $19^{+}$cells from healthy donors and $\mathrm{CD}_{138^{+}}$primary cells from MM patients were obtained by MACS selection (Miltenyi Biotec). Peripheral blood NK (PB-NK) from healthy individuals and NK from MM patients (MM-NK) were obtained after doing Ficoll in peripheral blood and afterwards MACS selection of NK cells (Miltenyi Biotec).

Luminescence and fluorescence in vivo imaging. Generation of green fluorescent protein-Firefly luciferase (GFP-FFLuc)-expressing ARP1 cell line for in vivo experiments was previously reported. ${ }^{20}$ Mice were anaesthetized and subjected to serial bioluminescence imaging as previously described ${ }^{20}$ using a Xenogen IVIS 200 system (Caliper, Waltham, MA, USA). After euthanizing the mice, tumor areas were visually inspected and then visualized also under a fluorescent stereo microscope Leica M2025 FA (Leica Microsystems Inc, Buffalo Grove, IL, USA). The Lymphoma/Myeloma Department gave us advice to set up the in vivo MM model using ARP1 cell line.

Cytotoxicity assays. Two different techniques were used to label target cells: either with ${ }^{51} \mathrm{Cr}$ (Amersham Pharmacia Biotech, Piscataway, NJ, USA) for $2 \mathrm{~h}$ or with BATDA reagent (Perkin-Elmer, Waltham, MA, USA) for $10 \mathrm{~min}$, then washed and added to effector cells (NK) in triplicate into 96-well round bottom plates (Corning, Corning, NY, USA) at different NK:target cell ratios. After $4 \mathrm{~h}$, supernatants were transferred either to a Luma-Plate-96 (Perkin-Elmer) for ${ }^{51} \mathrm{Cr}$ or 
to White flat bottom plates with Delfia Europium solution. ${ }^{51} \mathrm{Cr}$ or Batda-Europium release by target cells after lysis was measured either on a TOP Count NXT microplate scintillation and luminescence counter (Perkin-Elmer) for ${ }^{51} \mathrm{Cr}$ or in a fluorescence reader (Tecan, Männedorf, Switzerland). Cytotoxicity was determined by the formula: cytotoxicity $=($ sample value - spontaneous lysis $) /($ maximum lysis - spontaneous lysis) $\times 100 \%$. For blocking or inhibition assays, all reagents were added for $30 \mathrm{~min}$ at $37^{\circ} \mathrm{C}$ either to NK or target cells before the cytotoxicity assay. U18666A was added to $\mathrm{NK}$ for $2 \mathrm{~h}$ at $37^{\circ} \mathrm{C}$ previous to the cytotoxicity assay. Anti-NKG2D (clone 1498810) or anti-NKP30 (clone p30-15) were added to NK cells at $5 \mu \mathrm{g} / \mathrm{ml}$. GranzymeB inhibitor Z-AAD-CMK (Calbiochem, San Diego, CA, USA) was added to NK at $600 \mathrm{nM}$. Caspase-3 inhibitor Z-DEVD-FMK (BD Biosciences, San Jose, CA, USA) was added to target cells at $20 \mu \mathrm{M}$. Cathepsin inhibitor E64 (Sigma-Aldrich, St. Louis, MO, USA) was added to target cells at $10 \mu \mathrm{M}$. The lipid raft inhibitor Filipin III (Sigma-Aldrich) was added to NK cells. Cholesterol synthesis inhibitor U18666A (Sigma-Aldrich) was added to NK cells at $5 \mu \mathrm{g} / \mathrm{ml}$. The CXCL12-CXCR4 inhibitor AMD3100 (Sigma-Aldrich) was added at $40 \mu \mathrm{M}$. For calculation of cytotoxicity reduction after blocking NK receptors, inhibiting GrB and Caspase-3 pathway or adding Filipin III, U18666A, E64or AMD3100, values were calculated relative to control NK cells without blocking any receptor or without inhibiting any pathway.

Stromal cells and ELISAs. BM stromal cells (BMSC) from MM patients were obtained from BM aspirate after doing Ficoll to isolate mononuclear cells, expanded in vitro in DMEM $+10 \%$ FBS till they were $85 \%$ confluent and then the night before to the cytotoxicity assays they were added into 96-well plates at 10000 cells/well where the cytotoxicity assay was performed. CXCL12 levels in culture supernatant were measured with Human CXCL12/SDF-1 alpha Quantikine ELISA Kit (R\&D Systems, Minneapolis, MN, USA).

Confocal fluorescence microscopy. Target cells were stained with CellTracker Blue CMAC (7-amino-4-chloromethylcoumarin) and NK were stained with CellTracker Green Bodipy dye (8-Chloromethyl-4,4-Difluoro-1,3,5,7-Tetramethyl-4-Bora-3a,4a-Diaza-S-Indacene) (Invitrogen, Life Technologies, Eugene, NY, USA) for $30 \mathrm{~min}$ at $37^{\circ} \mathrm{C}$ in serum-free media. After washing, cells were mixed at a 1:1 ratio in 1.5-ml tubes, spun at low speed and allowed to co-culture in cell pellet at $37^{\circ} \mathrm{C}$ for 10,20 or $40 \mathrm{~min}$, depending on the experiment. For the 24-72 $\mathrm{h}$ experiments, cells were added into a 24-well plate at 1 million cells $/ \mathrm{ml}$ and then transferred onto microscope slides using a cell concentrator (Cytofuge 2, IRIS International, Chatsworth, CA, USA), fixed with 3\% methanol-free formaldehyde and then permeabilized. For immunostaining, primary antibodies were incubated for $30 \mathrm{~min}$ at $37^{\circ} \mathrm{C}$ and secondary antibodies were incubated for $20 \mathrm{~min}$ at $37^{\circ} \mathrm{C}$. To visualize CB-NK and MM cells with the stroma, BMSC from MM patients were added over microscope slides in well chambers the night before of the experiment to allow the stroma to adhere to the slides. Next day, CB-NK (stained in green with bodipy) and MM cells (stained in blue with CMAC) were added and allowed to co-culture for $40 \mathrm{~min}$, then slides were fixed, permeabilized and immunostained for actin (Phalloidin) as described above.

Primary antibodies used in confocal fluorescence microscopy were GrB-Alexa Fluor-647, NKP30-PE and NKP30-Alexa Fluor-647 from BD Biosciences, NKG2DAlexa Fluor-700 (R\&D Systems), EEA1, Rab7 (D95F2), Rab11 (D4F5) from Cell Signaling Technology (Boston, MA, USA), Phalloidin-Alexa Fluor-594 and Phalloidin-Alexa Fluor-647 from Invitrogen (Invitrogen, Life Technologies). Secondary antibody used was Anti-Rabbit IgG-Alexa Fluor-647 from Cell Signaling. For ROS and lysosome detection, Cell ROX Deep Red Reagent and Lyso Tracker Deep Red (Molecular Probes, Invitrogen, Life Technologies) were added into the media at $3 \mu \mathrm{M}$ and $75 \mathrm{nM}$, respectively.

Images were acquired using an Olympus IX81 microscope (Olympus Corporation, Center Valley, PA, USA). Image acquisition was performed using 405, 488 and 633 lasers for excitation, doing Z-stack acquisition images and applying the corresponding filters for each dye used. Image analysis was performed with the software FluoView Viewer FV-ASW version 04.00.02.09 (Olympus Corporation). To obtain fluorescence intensity values, four different views/slide were analyzed, quantifying $100 \mathrm{cells} / \mathrm{view}$. Numerical values obtained were then used to perform statistical analysis with SPSS statistics, version 19 (IBM, Chicago, IL, USA).

Time-lapse in vivo imaging. Time-lapse in vivo imaging was performed by staining target and effector cells with CMAC and Bodipy as described above. Cells were added together into an eight-well plate and imaged using the 405- and 488-nm wave length lasers for $2 \mathrm{~h}$ performing one image acquisition/minute.
Flow cytometry. To visualize tumor cells and CB-NK in the different tissues in mice by flow cytometry and degranulation of CB-NK in vitro, $10^{6} \mathrm{cells} / \mathrm{ml}$ were stained for $30 \mathrm{~min}$ at $4^{\circ} \mathrm{C}$. The following conjugated antibodies were used: NKG2D-PeCy7, CD16-PeCy5, CD138-APC, CD56-V450, CD56-PeCy5, and CD107a-V450 from BD Biosciences. Data were acquired by the Beckman Coulter Gallios device (Beckman Coulter, Inc., Brea, CA, USA). Analysis was performed using the FlowJo (Tree Star, Ashland, OR, USA) software.

FACS sorting. To FACS-sort ARP1 cells that had been primarily synapsed with CB-NK, ARP1 cells stained in blue with CMAC and CB-NK in green with Bodipy were allowed to co-culture in cell pellet for $40 \mathrm{~min}$. Then, propidium iodide was added at $3 \mu \mathrm{g} / \mathrm{ml}$ and cells were sorted in a BD FACSAria III cell sorter (BD Biosciences), based on FSC/SSC properties, single cells, viability (propidium iodide exclusion) and $\mathrm{CMAC}^{+}$positivity. Sorted cells were used afterwards for cytotoxicity assays and confocal fluorescence microscopy experiments.

Indirect cytotoxicity. For indirect cytotoxicity experiments, sorted ARP1 cells that had been co-cultured in cell pellet with CB-NK were added as effector cells in a cytotoxicity assay with fresh ARP1 cells (never exposed to CB-NK) as target cells at different effector:target cell ratios. As a negative control, ARP1 cells untouched by CB-NK were sorted and used in parallel also as effector cells. For the stroma experiment, in parallel ARP1 cells were added to a plate with BMSC from MM patients previously added the night before. Cytotoxicity assays were performed as described above.

Statistical analysis. Pearson's correlation test was performed to analyze correlation between MFI for NKG2D and disease progression in mice. Mann-Whitney $U$ test was used to analyze the comparison between groups for the confocal fluorescence images, flow cytometry and cytotoxicity assays. Paired $T$-test was performed to analyze the difference in the cytotoxicity assay for different groups in the individual experiments. Mann-Whitney $U$ test was used to analyze the comparison between different groups in cytotoxicity assays for the analysis of all different experiments together. All statistical analyses were performed using either SPSS statistics, version 19 or GraphPad Prism, version 6.02 (GraphPad Software, Inc., San Diego, CA, USA).

\section{Conflict of Interest}

The authors declare no conflict of interest.

Acknowledgements. We acknowledge David J McConkey for his advice in different studies, the Department of Cancer Systems Imaging microscopy core facility at MDACC (Houston, TX, USA) for equipment use and technical support. We acknowledge the multiple myeloma tumor bank from MDACC for providing patient samples and Mercedes Garayoa (Centro de Investigación del Cáncer, IBMCCUniversidad de Salamanca-CSIC, Spain) for providing bone marrow stromal cells from multiple myeloma patients; Dean A Lee and Laurence JN Cooper (MDACC) for providing K562-based antigen-presenting cells expressing membrane-bound IL-21 ('Clone 9.mblL21') for CB-NK expansion. SPORE grant provided financial support to the myeloma tumor bank. Katz Foundation and Institute of Health Carlos III (project: PI11/01090) provided funding for all NK studies. BMA was the recipient of a 'Sara Borrell' fellowship from Institute of Health Carlos III. CB is supported by a Miguel Servet II contracts from the FIS/ISCIII (CPII13/00011).

1. Bertaina A, Locatelli $F$, Moretta $L$. Transplantation and innate immunity: the lesson of natural killer cells. Ital J Pediatr 2009; 35: 44.

2. Masilamani M, Peruzzi G, Borrego F, Coligan JE. Endocytosis and intracellular trafficking of human natural killer cell receptors. Traffic 2009; 10: 1735-1744.

3. Lugini L, Cecchetti S, Huber V, Luciani F, Macchia G, Spadaro F et al. Immune surveillance properties of human NK cell-derived exosomes. J Immunol 2012; 189: 2833-2842.

4. Ewen CL, Kane KP, Bleackley RC. A quarter century of granzymes. Cell Death Differ 2012; 19: $28-35$.

5. Okada S, Li Q, Whitin JC, Clayberger C, Krensky AM. Intracellular mediators of granulysininduced cell death. J Immunol 2003; 171: 2556-2562.

6. Aporta A, Catalán E, Galán-Malo P, Ramírez-Labrada A, Pérez M, Azaceta G et al. Granulysin induces apoptotic cell death and cleavage of the autophagy regulator Atg5 in human hematological tumors. Biochem Pharmacol 2014; 87: 410-423. 
7. Saini RV, Wilson C, Finn MW, Wang T, Krensky AM, Clayberger C. Granulysin delivered by cytotoxic cells damages endoplasmic reticulum and activates caspase-7 in target cells. J Immunol 2011; 186: 3497-3504.

8. Zhang H, Zhong C, Shi L, Guo Y, Fan Z. Granulysin induces cathepsin B release from lysosomes of target tumor cells to attack mitochondria through processing of bid leading to necroptosis. J Immunol 2009; 182: 6993-7000.

9. Smith D, Yong K. Multiple myeloma. BMJ 2013; 346: $\{3863$.

10. Palumbo A, Anderson K. Multiple myeloma. N Engl J Med 2011; 364: 1046-1060.

11. Aronson LI, Davies FE. DangER: protein ovERload. Targeting protein degradation to treat myeloma. Haematologica 2012; 97: 1119-1130.

12. Sonneveld P, Goldschmidt H, Rosiñol L, Bladé J, Lahuerta JJ, Cavo M et al. Bortezomibbased versus nonbortezomib-based induction treatment before autologous stem-cell transplantation in patients with previously untreated multiple myeloma: a meta-analysis of phase III randomized, controlled trials. J Clin Oncol 2013; 31: 3279-3287.

13. Mimura N, Fulciniti M, Gorgun G, Tai YT, Cirstea D, Santo L et al. Blockade of XBP1 splicing by inhibition of IRE1alpha is a promising therapeutic option in multiple myeloma Blood 2012; 119: 5772-5781.

14. Tagoug I, Jordheim LP, Herveau S, Matera EL, Huber AL, Chettab K et al. Therapeutic enhancement of ER stress by insulin-like growth factor I sensitizes myeloma cells to proteasomal inhibitors. Clin Cancer Res 2013; 19: 3556-3566.

15. Wu WK, Wu YC, Yu L, Li ZJ, Sung JJ, Cho CH. Induction of autophagy by proteasome inhibitor is associated with proliferative arrest in colon cancer cells. Biochem Biophys Res Commun 2008; 374: 258-263.

16. Wang XJ, Yu J, Wong SH, Cheng AS, Chan FK, Ng SS et al. A novel crosstalk between two major protein degradation systems: regulation of proteasomal activity by autophagy. Autophagy 2013; 9: 1500-1508.

17. Choi AM, Ryter SW, Levine B. Autophagy in human health and disease. $N$ Engl J Med 2013; 368: $1845-1846$

18. Carroll RG, Martin SJ. Autophagy in multiple myeloma: what makes you stronger can also kill you. Cancer Cell 2013; 23: 425-426.

19. Milani M, Rzymski T, Mellor HR, Pike L, Bottini A, Generali D et al. The role of ATF4 stabilization and autophagy in resistance of breast cancer cells treated with Bortezomib. Cancer Res 2009; 69: 4415-4423.

20. Shah N, Martin-Antonio B, Yang H, Ku S, Lee DA, Cooper LJN et al. Antigen presenting cell-mediated expansion of human umbilical cord blood yields log-scale expansion of natural killer cells with anti-myeloma activity. PLoS One 2013; 8 : e76781.

21. Swift BE, Williams BA, Kosaka Y, Wang XH, Medin JA, Viswanathan S et al. Natural killer cell lines preferentially kill clonogenic multiple myeloma cells and decrease myeloma engraftment in a bioluminescent xenograft mouse model. Haematologica 2012; 97: 1020-1028.
22. Durymanov MO, Beletkaia EA, Ulasov AV, Khramtsov YV, Trusov GA, Rodichenko NS et al. Subcellular trafficking and transfection efficacy of polyethylenimine-polyethylene glycol polyplex nanoparticles with a ligand to melanocortin receptor-1. J Control Release 2012; 163 : 211-219.

23. Maxfield FR, McGraw TE. Endocytic recycling. Nat Rev Mol Cell Biol 2004; 5: 121-132.

24. Peruzzi G, Masilamani M, Borrego $F$, Coligan JE. Endocytosis as a mechanism of regulating natural killer cell function: unique endocytic and trafficking pathway for CD94/NKG2A. Immunol Res 2009; 43: 210-222.

25. Wayteck L. Effects of endocytosis inhibitors on transfection mediated by polyplexes and lipoplexes carrying mRNA. Master thesis, Ghent University, Ghent, Belgium, 2010.

26. Koh $\mathrm{CH}$, Cheung NS. Cellular mechanism of U18666A-mediated apoptosis in cultured murine cortical neurons: bridging Niemann-Pick disease type $C$ and Alzheimer's disease. Cell Signal 2006; 18: 1844-1853.

27. Liu D, Martina JA, Wu XS, Hammer JA 3rd, Long EO. Two modes of lytic granule fusion during degranulation by natural killer cells. Immunol Cell Biol 2011; 89: 728-738.

28. Martín-Antonio B, Carmona M, Falantes J, Gil E, Baez A, Suarez M et al. Impact of constitutional polymorphisms in VCAM1 and CD44 on CD34 + cell collection yield after administration of granulocyte colony-stimulating factor to healthy donors. Haematologica 2011; 96: 102-109.

29. Aits S, Jäättelä M. Lysosomal cell death at a glance. J Cell Sci. 2013; 126(Pt 9): 1905-1912.

30. Bröker LE, Kruyt FA, Giaccone G. Cell death independent of caspases: a review. Clin Cancer Res 2005; 11: 3155-3162.

31. Corrado C, Raimondo S, Chiesi A, Ciccia F, De Leo G, Alessandro R. Exosomes as intercellular signaling organelles involved in health and disease: basic science and clinical applications. Int J Mol Sci 2013; 14: 5338-5366.

32. Saka HA, Valdivia R. Emerging roles for lipid droplets in immunity and host-pathogen interactions. Annu Rev Cell Dev Biol 2012; 28: 411-437.

33. Maxfield FR, Tabas I. Role of cholesterol and lipid organization in disease. Nature 2005; 438: 612-621.

34. Carnero A. MAP17, a ROS-dependent oncogene. Front Oncol 2012; 2: 112

35. Garg AD, Dudek AM, Ferreira GB, Verfaillie T, Vandenabeele P, Krysko DV et al. ROS-induced autophagy in cancer cells assists in evasion from determinants of immunogenic cell death. Autophagy 2013; 9: 1292-1307.

36. Lamy L, Ngo VN, Emre NC, Shaffer AL 3rd, Yang Y, Tian E et al. Control of autophagic cell death by caspase-10 in multiple myeloma. Cancer Cell 2013; 23: 435-449.

37. Appelqvist $\mathrm{H}$, Wäster $\mathrm{P}$, Kågedal $\mathrm{K}$, Öllinger $\mathrm{K}$. The lysosome: from waste bag to potential therapeutic target. J Mol Cell Biol 2013; 5: 214-226.

38. Huang $\mathrm{CC}$, Chen $\mathrm{KL}$, Cheung $\mathrm{CH}$, Chang JY. Autophagy induced by cathepsin S inhibition induces early ROS production, oxidative DNA damage, and cell death via xanthine oxidase. Free Radic Biol Med 2013; 65: 1473-1486.

Supplementary Information accompanies this paper on Cell Death and Differentiation website (http://www.nature.com/cdd) 